\title{
Grand challenges for evolutionary robotics
}

\author{
Agoston E. Eiben* \\ Department of Computer Science, VU University, Amsterdam, Netherlands \\ *Correspondence: a.e.eiben@vu.nl \\ Edited and reviewed by: \\ Stefano Nolfi, CNR, Italy
}

Keywords: evolutionary computing, evolutionary design, embodiment, self-reproduction, open-ended evolution, on-line evolution

Evolutionary Robotics is a field that "aims to apply evolutionary computation techniques to evolve the overall design or controllers, or both, for real and simulated autonomous robots" (Vargas et al., 2014). This approach is "useful both for investigating the design space of robotic applications and for testing scientific hypotheses of biological mechanisms and processes" (Floreano et al., 2008). However, as noted in Bongard (2013) "the use of metaheuristics (i.e., evolution) sets this subfield of robotics apart from the mainstream of robotics research," which "aims to continuously generate better behavior for a given robot, while the long-term goal of Evolutionary Robotics is to create general, robot-generating algorithms."

One could say that Evolutionary Robotics is a test ground or experimental toolbox to study various issues arising on the road to intelligent and autonomous machines. The related issues include embodied cognition and intelligence, self-organization and collective behavior, the emergence of communication and cooperation, co-evolution, neuro-evolution, and many more with robots forming the substrate or medium for the experiments (Nolfi and Floreano, 2000; Wang et al., 2006; Floreano et al., 2008; Trianni, 2008; Doncieux et al., 2011; Bongard, 2013; Vargas et al., 2014). Given the fact that many Evolutionary Robotics investigations are performed in simulation, there is a big overlap with a subfield in Artificial Life research that is concerned with evolving virtual creatures and societies. However, I think it is safe to say that robotics can be distinguished because it ultimately aims at real physical robots (a.k.a. intelligent machines, animate artifacts, or artificial organisms) that exist and operate in the real world. Their bodies can be made of traditional mechatronic components, (self-) assembled from simple modular units, formed by some soft material, 3D printed plastics, some fancy new stuff invented by material scientists, or any combination of these, but in the end the robots must be physical entities. Therefore, Grand Challenges for Evolutionary Robotics must be tangible. Furthermore, Grand Challenges should be demonstrations of evolution, either a particular property of it, or the process of evolution as a whole.

In the following, I propose three Grand Challenges, subject to discussion. This list is not meant to capture the ultimate goals for the field. Rather, it is meant to inspire the community to deliberate and collectively identify the bold dreams that can lead further developments. This paper will have achieved its main goal if the list of Grand Challenges is discussed and revised, leading to an adjusted version that has a broad support.

\section{THE ROBOT KANGAROO}

Natural and artificial evolution are praised for the ability to "think" out of the box. The field of evolutionary computing and evolutionary design has demonstrated that artificial evolution can deliver solutions that humans find unexpected and original (Bentley and Corne, 2002; Eiben and Smith, 2003). Further to being cool, such solutions can advance science and engineering because, in principle, they can be analyzed and reverse engineered. Understanding why and how they work can provide new insights, design guidelines, or adjustment to the current theory. As for natural evolution, consider the kangaroo. For thousands of years, people have been drawing odd animals, like chimeras and dragons. However, these were most often combinations and/or exaggerations of existing animals: strange - yes, original - no. In the meanwhile, before the discovery of Australia no one had imagined an animal with a pouch. Thus, the kangaroo is a metaphor for the truly original designs evolution can come up with. The challenge dubbed The Robot Kangaroo is to deliver the promise of evolution to be original in a robotics context. That is, to demonstrate an evolved robot whose design (morphology and/or control system, and/or behavior) is surprising. Clearly, it is hard, if not impossible, to pre-define surprising and it could be argued that it is too subjective to be useful. However, the same can be said about beautiful, but this does not prevent Miss Universe contests from being organized. To put it pragmatically: Original design? We can't define it, but we'll know it when we see it.

\section{SELF-REPRODUCING ROBOTS THAT EVOLVE IN REAL TIME AND REAL SPACE}

Evolutionary Computing is, well, computing. Producing a new individual in an evolving population is just a matter of creating a new piece of digital code. The same holds for evolving virtual creatures in Artificial Life and Evolutionary Robotics experiments conducted in simulation. However, as noted in Fernando et al. (2011), such systems seriously lack "the richness of matter that is a source of challenges and opportunities not yet matched in artificial algorithms." Going from digital evolutionary systems to physical ones will be a game changer in several ways and will represent a second major transition from a historical perspective (Eiben et al., 2012). The first transition took place in the twentieth century when computers made it possible to create, study, and utilize artificial evolutionary processes in imaginary, digital spaces. This was a transition from evolution in wetware to evolution in software. In the twenty-first century, we could make a transition from evolution in software to evolution in hardware. The corresponding Grand Challenge entails that robots can physically (self-)reproduce, that is, it 
requires an asexual or sexual reproduction mechanism that can create a new physical robot based on genetic information of one or two existing robots (Eiben et al., 2013). The new kind of Evolutionary Robotics will switch from simulations with occasional fitness evaluations in real hardware to the physical world with occasional use of (internal) simulators. In such a setting, we can close the reality gap and switch from (ab)using evolution as an off-line optimizer to employing it as a force for on-line adaptation. The resulting systems will exhibit more realistic evolutionary processes with the inherent noise, uncertainty, and richness of physical interactions. Furthermore, in such systems we can investigate the co-evolution of minds and bodies and study their interactions both ways, not only study how the body shapes the mind, but also how the mind shapes the body and how their emergent interactions lead to interesting behaviors (Pfeifer and Bongard, 2006). It is very likely that the evolutionary dynamics and the outcomes will be different from those in simulation. The bottom line is: Matter matters.

\section{OPEN-ENDED ROBOT EVOLUTION}

Evolutionary Robotics can contribute to engineering as well as to biology (Waibel et al., 2011, Long, 2012). When using it for engineering purposes or studies in Artificial Intelligence and Artificial Life, there are no restrictions on the evolutionary mechanisms. The system does not need to be biologically feasible - anything goes as long as it delivers good designs and interesting behaviors. On the other hand, a biologically plausible Evolutionary Robotics system represents a physical (not digital) model of evolution, hence it can be used to conduct scientific studies into the principles of biological evolution. As of today, the level of technology will not allow for a complete emulation of all underlying chemical and biological micro-mechanisms in some artificial substrate. However, even a system that mimics the macro-mechanisms (e.g., selection, reproduction, and heredity) in a physical medium is a great leap forward from the purely software-based research tools. Even if such a system is just an approximation of the biological reality, at least it will not violate the laws of physics and will be able to exploit the richness of matter. The third Grand Challenge is to build a real world evolutionary system of physical robots that undergo open-ended evolution in an open environment. One important property of such a system is the type of selection driving it. Selection can be purely environmental without a userimposed, quantifiable fitness function pursuing only viability, but in principle, this can be extended with mechanisms that represent preferences of the user/experimenter also aiming at some utility (Haasdijk et al., 2014). For a maximum match with biological evolution, the system should be driven by environmental selection only. Given enough time, this system will hopefully evolve and adapt to the environment. With such a system, we could gain insights into fundamental issues, e.g., the minimal conditions for evolution to take place, the factors influencing evolvability, or the rate of progress under various circumstances. Perhaps we will witness some of the events natural evolution encountered, such as the emergence of species, maybe even the Cambrian explosion. When achieved, this Grand Challenge could bridge Biology, Evolutionary Robotics, and Artificial Life producing a new category: Life, but not as we know it.

\section{ACKNOWLEDGMENTS}

I would like to thank Nicolas Bredeche, Stephane Doncieux, and Jean-Baptiste Mouret for the inspiring discussions that shaped my views reflected in this paper.

\section{REFERENCES}

Bentley, P., and Corne, D. (2002). Creative Evolutionary Systems. San Francisco: Morgan Kaufmann.

Bongard, J. (2013). Evolutionary robotics. Commun. ACM 56, 74-85. doi:10.1145/2492007.2493883

Doncieux, S., Bredeche, N., and Mouret, J.-B. (eds) (2011). New Horizons in Evolutionary Robotics, Volume 341 of Studies in Computational Intelligence. Berlin Heidelberg: Springer-Verlag.

Eiben, A., Bredeche, N., Hoogendoorn, M., Stradner, J., Timmis, J., Tyrrell, A., et al. (2013). “The triangle of life: evolving robots in real-time and real-space," in Advances in Artificial Life, ECAL 2013, eds P. Liò, O. Miglino, G. Nicosia, S. Nolfi, and M. Pavone (Cambridge, MA: MIT Press), 1056-1063.

Eiben, A. E., Kernbach, S., and Haasdijk, E. (2012). Embodied artificial evolution - artificial evolutionary systems in the 21st century. Evol. Intell. 5, 261-272. doi:10.1007/s12065-012-0071-x

Eiben, A. E., and Smith, J. (2003). Introduction to Evolutionary Computing. Berlin Heidelberg: SpringerVerlag.

Fernando, C., Kampis, G., and Szathmáry, E. (2011). Evolvability of natural and artificial systems. Procedia Comput. Sci. 7, 73-76. doi:10.1016/j.procs.2011. 12.023

Floreano, D., Husbands, P., and Nolfi, S. (2008). "Evolutionary robotics," in Springer Handbook of Robotics, Vol. G.61, eds B. Siciliano and O. Khatib (Springer), 1423-1451.

Haasdijk, E., Bredeche, N., and Eiben, A. E. (2014). Combining environment-driven adaptation and task-driven optimisation in evolutionary robotics. PLoS ONE, 9:e98466. doi:10.1371/journal.pone. 0098466

Long, J. (2012). Darwin's Devices: What Evolving Robots Can Teach Us About the History of Life and the Future of Technology. New York: Basic Books.

Nolfi, S., and Floreano, D. (2000). Evolutionary Robotics: The Biology, Intelligence, and Technology of SelfOrganizing Machines. Cambridge, MA: MIT Press.

Pfeifer, R., and Bongard, J. (2006). How the Body Shapes the Way We Think. Cambridge, MA: MIT Press.

Trianni, V. (2008). Evolutionary Swarm Robotics Evolving Self-Organising Behaviours in Groups of Autonomous Robots, Volume 108 of Studies in Computational Intelligence. Springer.

Vargas, P., Paolo, E. D., Harvey, I., and Husbands, P. (eds) (2014). The Horizons of Evolutionary Robotics. Cambridge, MA: MIT Press.

Waibel, M., Floreano, D., and Keller, L. (2011). A quantitative test of Hamilton's rule for the evolution of altruism. PLOS Biology 9:e1000615. doi:10.1371/ journal.pbio.1000615

Wang, L., Tan, K., and Chew, C. (2006). Evolutionary Robotics: From Algorithms to Implementations, Volume 28 of World Scientific Series in Robotics and Intelligent Systems. Singapore: World Scientific Publishing.

Conflict of Interest Statement: The author declares that the research was conducted in the absence of any commercial or financial relationships that could be construed as a potential conflict of interest.

Received: 23 May 2014; accepted:06 June 2014; published online: 30 June 2014.

Citation: Eiben AE (2014) Grand challenges for evolutionary robotics. Front. Robot. AI 1:4. doi: 10.3389/frobt.2014.00004

This article was submitted to Evolutionary Robotics, a section of the journal Frontiers in Robotics and AI. Copyright (c) 2014 Eiben. This is an open-access article distributed under the terms of the Creative Commons Attribution License (CC BY). The use, distribution or reproduction in other forums is permitted, provided the original author(s) or licensor are credited and that the original publication in this journal is cited, in accordance with accepted academic practice. No use, distribution or reproduction is permitted which does not comply with these terms. 